\title{
RISK ANALYSIS - AN INSTRUMENT FOR TECHNOLOGY SELECTION
}

\author{
IVAN POPCheV* AND IRINA RADEVA \\ Institute of Information and Communication Technologies, \\ Bulgarian Academy of Sciences, \\ Acad. Georgy Bonchev St., Bl. 2, 1113 Sofia, \\ e-mails: ipopchev@iit.bas.bg; iradeva@iit.bas.bg
}

\begin{abstract}
The article is an attempt to summarize the schematic picture of negative and undefined impacts of new technologies implementations combined in the formula Industry 4.0. The aim is to identify potential risks of using technologies and which of them are most risky. Major technologies have been analysed by their unknown and negative impacts. The variety of risks are introduced in eight generalized risk categories concerning problems of privacy and data security, change in labour market, fragmentation, responsibility and accountability, ecology, ecosystems and ethics, changes in income/cost structure and ownership of assets. The technologies are selected by using quasi-multicriteria analysis SIGMA. The results could be used as a framework, starting point to deeper understanding of dimensions, application and impacts of technologies.

Keywords: risk analysis, quasi multicriteria analysis, Industry 4.0 - technology, selection.
\end{abstract}

\section{INTRODUCTION}

The concept of "Industry 4.0" as a new platform provides wide opportunities for digitalization of assets and economic agents' activities included in a value chain and their integration into digital ecosystem. Prerequisites for its realization are the transformation of leading IT technologies, known as "disruptive" $[1,2]$. The adaptation to the digital environment raises a number of issues as:

- First: clarification and systematization of the terminology: "digital skills", "digital literacy", "digital competence", "digital divide", etc. [3];

\footnotetext{
* Corresponding author.

DOI: 10.7546/EngSci.LVI.19.04.01
}

(C) Инженерни науки, год. LVI, 2019, № $4 \quad 5$

(C) Engineering Sciences, LVI, 2019, No. 4 
- Second: classification of digital skills and identifying the specifics of structure and formation of such skills in professional and hierarchical positions [4];

- Third: developing targeted programs that promote adaptation to the digital environment [5];

- Forth: identifying and analysing risks in digital technologies.

The latter problem is particularly relevant. The Industry 4.0 poses risks that are not yet known. Without adequate professional training, the individual becomes the "narrow" place in the relationship "human - digital environment". This is a new paradigm in the search for strategic and tactical far-sighted decisions. It requires a fast and adequate solidarity response of involved parties in the processes. The resulting gap between technological opportunities and public, administrative and professional training at all levels of value chains requires maintaining social stability in labour markets and smoother transformation of the economy. In the long run, risk analysis measures have a greater chance as additional time allows for innovation and organizational change to work against the different types of risks. The increasing complexity and interdependence of technologies is accompanied by a significant increase in risk factors combined with not always adequate means of providing information security.

The article is an attempt to summarize the schematic picture of the analysis of the negative and undefined impacts of the implementation of new technologies, the results of which are combined by the formula of the Industry 4.0. The aim is to identify the potential risks of using technologies, as well as which of them are most risky. Major technologies have been analysed by their unknown and negative impacts according to the reported research $[6,7]$. The variety of risk effects are introduced as eight generalized risk categories.

\section{RISK EFFECTS AND EXPOSURES IN INDUSTRY 4.0}

The variety of risks is generalized in risk categories given in Table 1.

There are introduced following notifications of technologies: $\mathrm{S}_{\operatorname{Im} T}-\mathrm{im}-$ plantable technologies, $S_{D P}$ - our digital presence, $S_{V n I}$ - vision as the new interface, $\mathrm{S}_{\mathrm{WI}}$ - wearable internet, $\mathrm{S}_{\mathrm{UC}}$ - ubiquitous computing, $\mathrm{S}_{\mathrm{SoP}}$ - supercomputer in your pocket, $\mathrm{S}_{\mathrm{WoA}}$ - storage for all; $\mathrm{S}_{\mathrm{IoT}}$ - the Internet of things, $\mathrm{S}_{\mathrm{CH}}$ - the connected home, $\mathrm{S}_{\mathrm{SC}}$ - smart cities, $\mathrm{S}_{\mathrm{BDD}}$ - big data for decisions, $\mathrm{S}_{\mathrm{DC}}-$ driverless cars, $\mathrm{S}_{\mathrm{AIDM}}-\mathrm{AI}$ and Decision-Making, $\mathrm{S}_{\mathrm{AIWCJ}}-\mathrm{AI}$ and white-collar jobs, $\mathrm{S}_{\mathrm{R} \& \mathrm{~S}}$ - robotics and services, $\mathrm{S}_{\mathrm{SE}}$ - the sharing economy, $\mathrm{S}_{3 \mathrm{DP}}-3 \mathrm{D}$ printing in manufacturing, human health, and consumer products.

(c) Инженерни науки, год. LVI, 2019, № $4 \quad 6 \quad$ (c) Engineering Sciences, LVI, 2019, No. 4 
TABLE 1. Risk categories

\begin{tabular}{|l|}
\hline Risk category \\
\hline Privacy and data security $\left(\mathrm{R}_{\mathrm{D}}\right)$ \\
\hline Change in labour market $\left(\mathrm{R}_{\mathrm{L}}\right)$ \\
\hline Mental distraction $\left(\mathrm{R}_{\mathrm{P}}\right)$ \\
\hline Manipulation and echo camera $\left(\mathrm{R}_{\mathrm{M}}\right)$ \\
\hline Fragmentation $\left(\mathrm{R}_{\mathrm{F}}\right)$ \\
\hline Responsibility and accountability $\left(\mathrm{R}_{\mathrm{A}}\right)$ \\
\hline Ecology, ecosystems and ethics $\left(\mathrm{R}_{\mathrm{E}}\right)$ \\
\hline Changes in income/cost structure and ownership of assets $\left(\mathrm{R}_{\mathrm{S}}\right)$ \\
\hline
\end{tabular}

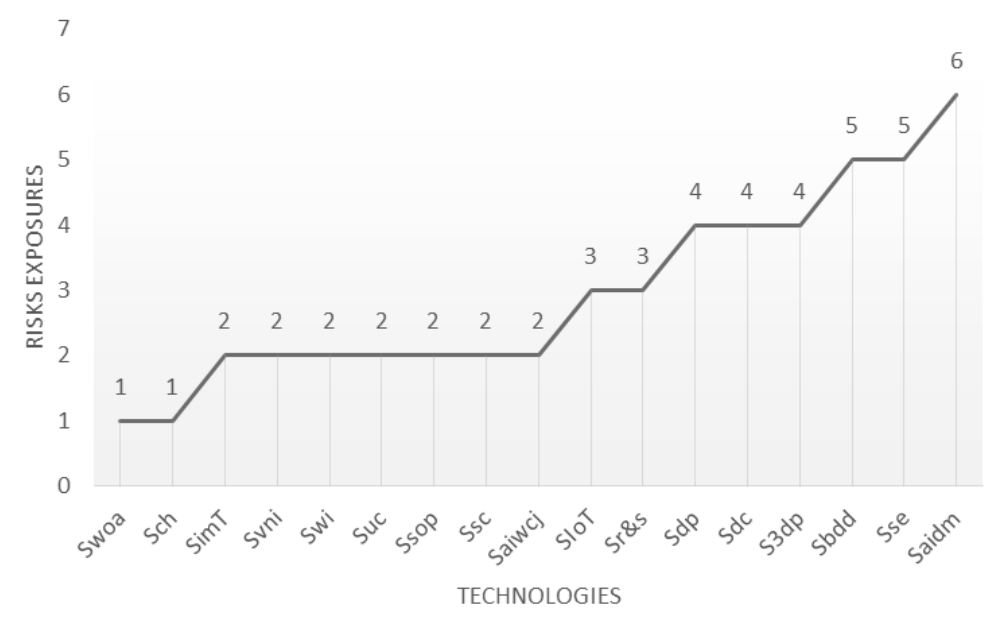

Fig. 1

Figure 1 shows the number of risks corresponding to technologies. Those at greatest number of risks are technologies: $\mathrm{S}_{\mathrm{AIDM}}-\mathrm{AI}$ and Decision-Making, $\mathrm{S}_{\mathrm{BDD}}$ - Big Data for Decisions, $\mathrm{S}_{\mathrm{SE}}-$ The Sharing Economy, $\mathrm{S}_{\mathrm{DP}}$ - Our Digital Presence, $\mathrm{S}_{\mathrm{DC}}-$ Driverless Cars and $\mathrm{S}_{3 \mathrm{DP}}-3 \mathrm{D}$ Printing in Manufacturing, Consumer Products and Human Health.

Undoubtedly, $\mathrm{S}_{\text {AIDM }}$ - artificial intelligence and decision - making is the "heart" of "disruptive technologies" because the related scientific breakthroughs form directions whose functioning depends to the greatest extent on the proper presentation of knowledge and imitation of human reasoning ability in decisionmaking [6]. The chart presented as a new research output should be the basis for specific strategic and operational risk analysis solutions for organizations and individuals.

(c) Инженерни науки, год. LVI, 2019, № $4 \quad 7 \quad$ (c) Engineering Sciences, LVI, 2019, No. 4 
The risk analysis problem involves engaging different resources and, above all, knowledge, experience and coordinated, at different levels, priority new research and development, subordinated to the problem of adaptation in the human-digital relationship. The growing divide in this relationship implies active action because of the rapid changes occurring in the current hyperconnected, more complex and more fragmented world than ever before.

\section{EVALUATING THE CUMULATIVE IMPACT OF THE RISKS}

If technologies are evaluated from a risk point of view, a well-known, simple but effective algorithm for quasi-multicriteria analysis SIGMA based on the degree of scattering of a discrete random variable around its mathematical expectation can be applied [8] with additional verification for complete cancellation of risk.

When designing the algorithm SIGMA are defined: $A$ - a finite set of alternative technologies; $S$ - a finite set of tested risk exposures; $\left\|a_{i j}\right\|$ evaluation matrix of the $i^{\text {th }}$ technological shifts $(i=1, \ldots m)$ in the $j^{\text {th }}$ risk exposures; $q_{j}$ - probability of occurrence of $j^{\text {th }}$ risk, $\forall j\left(q_{j}>0\right), \sum_{j=1}^{n} q_{j}=1$.

As an interactive procedure and a boundary case, it is acceptable to predict a situation where all probabilities are equal $q^{L}=\frac{1}{n}$ under the principle of Laplace (principle of insufficient reason). For each alternative $A_{i}$ are calculated: $\bar{a}_{i}=\sum_{j=1}^{n} q_{j} a_{i j}$, respectively $\bar{a}_{i}^{L}=\sum_{j=1}^{n} \frac{a_{i j}}{n}$ and mean square deviation $\sigma_{i}=\sqrt{\sum_{j=1}^{n}\left(a_{i j}-\bar{a}_{i}\right)^{2} q_{j}}$, respectively $\sigma_{i}^{L}=\sqrt{\sum_{j=1}^{n}\left(a_{i j}-\bar{a}_{i}^{L}\right)^{2} \frac{1}{n}}$ under Laplace.

For instance, the Decision Maker (DM) suggests to include in risk analysis ten criteria for selection of alternative, including two additional criteria $\left(K_{0}^{*}, K_{0}^{* L}\right)$. The formulated criteria could be different. One suggestive list is in Table 2.

The aim of the study is to evaluate which of the new ITs are riskiest and give recommendation for caution in their use, depending on the preferences of DM. Due to the fact that no quantitative risk assessments are not available, the only information on whether a technology is exposed to the relevant risk or not, the study is performed by following assumptions:

- 8 - risk factors;

(c) Инженерни науки, год. LVI, 2019, № 4

8

(C) Engineering Sciences, LVI, 2019, No. 4 
TABLE 2

\begin{tabular}{|l|l|l|}
\hline Criteria & DM Risk aversion & \\
\hline$K_{\delta}$ & Indifference to risk & $K_{\delta}=\max _{i} \bar{a}_{i}$ \\
\hline$K_{P}$ & Tendency to risk & $K_{P}=\max _{i}\left(\bar{a}_{i}+\sigma_{i}\right)$ \\
\hline$K_{C}$ & Caution to risk & $K_{C}=\max _{i}\left(\bar{a}_{i}-\sigma_{i}\right)$ \\
\hline$K_{O}$ & Full risk aversion & $K_{O}=\max _{i}\left(\bar{a}_{i}-2 \sigma_{i}\right)$ \\
\hline$K_{\delta}^{L}$ & Indifference to risk (Laplace) & $K_{\delta}^{L}=\max _{i} \bar{a}_{i}^{L}$ \\
\hline$K_{P}^{L}$ & Tendency to risk (Laplace) & $K_{P}^{L}=\max _{i}\left(\bar{a}_{i}^{L}+\sigma_{i}^{L}\right)$ \\
\hline$K_{C}^{L}$ & Caution to risk (Laplace) & $K_{C}^{L}=\max _{i}\left(\bar{a}_{i}^{L}-\sigma_{i}^{L}\right)$ \\
\hline$K_{O}^{L}$ & Full risk aversion (Laplace) & $K_{O}^{L}=\max _{i}\left(\bar{a}_{i}^{L}-2 \sigma_{i}^{L}\right)$ \\
\hline$K_{0}^{*}$ & Additional risk cancellation & $K_{0}^{*}=\max _{i}\left(\bar{a}_{i}^{L}-10 \sigma_{i}\right)$ \\
\hline$K_{0}^{* L}$ & Additional risk cancellation (Laplace) & $K_{0}^{* L}=\max _{i}\left(\bar{a}_{i}^{L}-10 \sigma_{i}^{L}\right)$ \\
\hline
\end{tabular}

- 17 - technologies tested;

- The maximum number of risks for one technology is 6 and the minimum is 1 (Fig. 1);

- The presence of risk is denoted by "1" and the absence by " 0 ";

- 12 - risk profiles (Table 3), each with an individual risk exposure;

TABLE 3

\begin{tabular}{|c|c|c|c|c|c|c|c|c|}
\hline $\begin{array}{cc}\text { Technology }_{\mathrm{i}} & \text { Risk }_{\mathrm{j}} \\
\end{array}$ & $\mathrm{R}_{\mathrm{D}}$ & $\mathrm{R}_{\mathrm{L}}$ & $\mathrm{R}_{\mathrm{P}}$ & $\mathrm{R}_{\mathrm{M}}$ & $\mathrm{R}_{\mathrm{F}}$ & $\mathrm{R}_{\mathrm{A}}$ & $\mathrm{R}_{\mathrm{E}}$ & $\mathrm{R}_{\mathrm{S}}$ \\
\hline $\mathrm{S}_{\mathrm{VNI}}, \mathrm{S}_{\mathrm{WI}}, \mathrm{S}_{\mathrm{IMT}}$ & 1 & 0 & 1 & 0 & 0 & 0 & 0 & 0 \\
\hline $\mathrm{S}_{\mathrm{DP}}$ & 1 & 0 & 0 & 1 & 1 & 0 & 1 & 0 \\
\hline$\overline{\mathrm{S}_{\mathrm{UC}}, \mathrm{S}_{\mathrm{SOP}}}$ & 0 & 0 & 0 & 1 & $\overline{1}$ & 0 & 0 & 0 \\
\hline $\mathrm{S}_{\mathrm{WOA}}, \mathrm{S}_{\mathrm{CH}}$ & 1 & 0 & 0 & 0 & 0 & $\overline{0}$ & 0 & 0 \\
\hline $\mathrm{S}_{\mathrm{IOT}}$ & 0 & 1 & 0 & 1 & 0 & 0 & 0 & 0 \\
\hline $\mathrm{S}_{\mathrm{SC}}$ & 1 & 0 & 0 & 0 & 0 & 0 & 1 & 0 \\
\hline $\mathrm{S}_{\mathrm{BDD}}, \mathrm{S}_{\mathrm{AIDM}}$ & 1 & 1 & 0 & 1 & 1 & 1 & 1 & 0 \\
\hline $\mathrm{S}_{\mathrm{DC}}$ & 1 & 1 & 0 & 0 & 1 & 0 & 0 & 1 \\
\hline $\mathrm{S}_{\text {AIWCJ }}$ & 0 & 1 & 0 & 0 & 0 & 1 & 0 & 0 \\
\hline $\mathrm{S}_{\mathrm{R} \& \mathrm{~S}}$ & 1 & 1 & 0 & 0 & 0 & 1 & 0 & 0 \\
\hline $\mathrm{S}_{\mathrm{SE}}$ & 1 & 1 & 0 & 1 & 0 & 1 & 0 & 1 \\
\hline $\mathrm{S}_{3 \mathrm{DP}}$ & 1 & 1 & 0 & 1 & 0 & 0 & 1 & 1 \\
\hline$q^{L}$ & 0.125 & 0.125 & 0.125 & 0.125 & 0.125 & 0.125 & 0.125 & 0.125 \\
\hline & 0.080 & 0.150 & 0.150 & 0.100 & 0.175 & 0.250 & 0.070 & 0.025 \\
\hline
\end{tabular}

(c) Инженерни науки, год. LVI, 2019, № 49

(c) Engineering Sciences, LVI, 2019, No. 4 
- The impact of each risk is assessed individually;

- The preferences of the DM are expressed by weight coefficients.

The result should be interpreted as a recommendation for attention (or avoidance) of a technology, depending on the risk tolerance of the DM.

Table 3 shows the technologies grouped by risk profiles, the corresponding risk exposures and the values of the weighting coefficients $q_{j}\left(R_{j}\right)$. The last line includes sample weights $q_{j}$ showing one variant of analysis and identification of the riskiest technologies, according to the preferences of the DM.

There are performed nine experiments for each risk category and scores are presented in Tables $4-11$.

\section{4. $R_{D}$ - PRIVACY AND DATA SECURITY}

Category $R_{D}$ includes risks: privacy/potential surveillance, online bullying/stalking, decreased data security, hacking, security threat (e.g. utility grid), cyberattacks, crime, vulnerability to cyberattacks, cyber risk, opportunity for short-term abuse of trust, identity theft.

The analysis of the results of the $\mathrm{R}_{\mathrm{D}}$ assessment shows:

TABLE $4 . \mathrm{R}_{\mathrm{D}}$ - Privacy and data security

\begin{tabular}{|c|c|c|c|c|c|c|c|c|c|c|}
\hline & $K_{\delta}$ & $K_{\delta}^{L}$ & $K_{P}$ & $K_{C}$ & $K_{O}$ & $K_{P}^{L}$ & $K_{C}^{L}$ & $K_{O}^{L}$ & $K_{0}^{*}$ & $K_{0}^{* L}$ \\
\hline $\begin{array}{c}\mathrm{S}_{\mathrm{VNI}}, \\
\mathrm{S}_{\mathrm{WI}} \\
\mathrm{S}_{\mathrm{IMT}}\end{array}$ & $\mathbf{1 . 0 0}$ & 0.25 & 1.75 & 0.25 & -0.50 & 0.68 & -0.18 & -0.62 & -6.50 & -4.08 \\
\hline $\mathrm{S}_{\mathrm{DP}}$ & $\mathbf{1 . 0 0}$ & 0.50 & 1.50 & 0.50 & 0.00 & 1.00 & 0.00 & -0.50 & -4.00 & -4.50 \\
\hline $\begin{array}{c}\mathrm{S}_{\mathrm{UC}}, \\
\mathrm{S}_{\mathrm{SOP}}\end{array}$ & 0.00 & 0.25 & 0.25 & -0.25 & -0.50 & 0.68 & -0.18 & -0.62 & -2.50 & -4.08 \\
\hline $\begin{array}{c}\mathrm{S}_{\mathrm{WOA}}, \\
\mathrm{S}_{\mathrm{CH}}\end{array}$ & $\mathbf{1 . 0 0}$ & 0.13 & $\mathbf{1 . 8 8}$ & 0.13 & -0.75 & 0.46 & -0.21 & -0.54 & -7.75 & -3.18 \\
\hline $\mathrm{S}_{\mathrm{IOT}}$ & 0.00 & 0.25 & 0.25 & -0.25 & -0.50 & 0.68 & -0.18 & -0.62 & -2.50 & -4.08 \\
\hline $\mathrm{S}_{\mathrm{SC}}$ & $\mathbf{1 . 0 0}$ & 0.25 & 1.75 & 0.25 & -0.50 & 0.68 & -0.18 & -0.62 & -6.50 & -4.08 \\
\hline $\begin{array}{c}\mathrm{S}_{\mathrm{BDD}}, \\
\mathrm{S}_{\mathrm{AIDM}}\end{array}$ & $\mathbf{1 . 0 0}$ & $\mathbf{0 . 7 5}$ & 1.25 & $\mathbf{0 . 7 5}$ & $\mathbf{0 . 5 0}$ & $\mathbf{1 . 1 8}$ & $\mathbf{0 . 3 2}$ & $-\mathbf{0 . 1 2}$ & $-\mathbf{1 . 5 0}$ & $-\mathbf{3 . 5 8}$ \\
\hline $\mathrm{S}_{\mathrm{DC}}$ & $\mathbf{1 . 0 0}$ & 0.50 & 1.50 & 0.50 & 0.00 & 1.00 & 0.00 & -0.50 & -4.00 & -4.50 \\
\hline $\mathrm{S}_{\mathrm{AIWCJ}}$ & 0.00 & 0.25 & 0.25 & -0.25 & -0.50 & 0.68 & -0.18 & -0.62 & -2.50 & -4.08 \\
\hline $\mathrm{S}_{\mathrm{R} \& \mathrm{~S}}$ & $\mathbf{1 . 0 0}$ & 0.38 & 1.63 & 0.38 & -0.25 & 0.86 & -0.11 & -0.59 & -5.25 & -4.47 \\
\hline $\mathrm{S}_{\mathrm{SE}}$ & $\mathbf{1 . 0 0}$ & 0.63 & 1.38 & 0.63 & 0.25 & 1.11 & 0.14 & -0.34 & -2.75 & -4.22 \\
\hline $\mathrm{S}_{3 \mathrm{DP}}$ & $\mathbf{1 . 0 0}$ & 0.63 & 1.38 & 0.63 & 0.25 & 1.11 & 0.14 & -0.34 & -2.75 & -4.22 \\
\hline
\end{tabular}

By criterion $K_{\delta}$ the maximum score is 1 . This indicates the presence of risk when using technology. Due to indifference to risk of this criteria are

(c) Инженерни науки, год. LVI, 2019, № 4 10 (c) Engineering Sciences, LVI, 2019, No. 4 
selected technologies $\mathrm{S}_{\mathrm{VNI}}, \mathrm{S}_{\mathrm{WI}}, \mathrm{S}_{\mathrm{IMT}}, \mathrm{S}_{\mathrm{DP}}, \mathrm{S}_{\mathrm{WOA}}, \mathrm{S}_{\mathrm{CH}}, \mathrm{S}_{\mathrm{SC}}, \mathrm{S}_{\mathrm{BDD}}, \mathrm{S}_{\mathrm{AIDM}}$, $\mathrm{S}_{D C}, \mathrm{~S}_{\mathrm{R} \& \mathrm{~S}}, \mathrm{~S}_{\mathrm{SE}}, \mathrm{S}_{3 D P}$ where this risk is presented (Table 4).

By criterion $K_{\delta}^{L}$ the maximum score is 0.75 . The choice is for $\mathrm{S}_{B D D}, \mathrm{~S}_{A I D M}$ technologies containing 6 out of 8 risks (these two technologies contain 6 risks and neither contains all 8). According to this criterion, DM preferences are by Laplace, i.e. $q^{L}=0.125$. The scores of all other Laplace criteria $K_{P}^{L}, K_{C}^{L}, K_{O}^{L}$ and $K_{0}^{* L}$ differ slightly. In all experiments, $\mathrm{S}_{\mathrm{BDD}}, \mathrm{S}_{\mathrm{AIDM}}$ technologies were rated as riskiest, i.e. not recommended, especially if DM prefers strictly avoid risk.

By criterion $K_{P}$ DM has tendency to risk. The maximum score is 1.88 . The choice to avoid using technology has been reduced to $\mathrm{S}_{\mathrm{WOA}}, \mathrm{S}_{\mathrm{CH}}$ which contain only $\mathrm{R}_{\mathrm{D}}$ risk. From an algorithm point of view, this is the only possible option because in the particular experiment other risks are not directly accounted. A similar choice has been made on the other two criteria $K_{C}$ - caution to risk and $K_{O}$ - full risk aversion.

The recommendations for avoiding technology based on risk preferences for DM only by the criteria $K_{\delta}, K_{P}, K_{C}, K_{O}$ and $K_{0}^{*}$ since the estimates $K_{\delta}^{L}$, $K_{P}^{L}, K_{C}^{L}, K_{O}^{L}$ and $K_{0}^{* L}$ are the same.

Specific experiments asses the riskiest technologies, i.e. in view of risk elimination, these technologies should be avoided.

\section{5. $\mathbf{R}_{\mathrm{L}}$ - CHANGE IN LABOR MARKET}

Category $\mathrm{R}_{\mathrm{L}}$ includes risks: Job losses, resilience after a job loss (because of less savings), contract/task-based labour (versus typically more stable longterm employment), global and regional supply and logistics chain: lower demand resulting in job losses, job automation, Day-to-day social norms, end of 9-to-5 and 24-hour services.

The analysis of the results in Table 5 for $\mathrm{R}_{\mathrm{L}}$ shows that by criterion $K_{\delta}$ again the maximum score of 1 due to the presence of this risk when using the technologies. Here, the DM is indifferent to the risk, selected technologies are $\mathrm{S}_{\mathrm{IOT}}, \mathrm{S}_{\mathrm{BDD}}, \mathrm{S}_{\mathrm{AIDM}}, \mathrm{S}_{\mathrm{DC}}, \mathrm{S}_{\mathrm{R} \& \mathrm{~S}}, \mathrm{~S}_{\mathrm{AIWCJ}}, \mathrm{S}_{\mathrm{SE}}, \mathrm{S}_{3 \mathrm{DP}}$

By criterion $K_{P}$, when DM has tendency to risk, the maximum score is 1,75. The choice to avoid using technology has been reduced to $\mathrm{S}_{\mathrm{IOT}}, \mathrm{S}_{\mathrm{AIWCJ}}$, which generally have two risks, one being $\mathrm{R}_{\mathrm{L}}$. The choice on other two criteria $K_{C}$ and $K_{O}$ recommends avoiding those at greatest number of risk exposures technologies $\mathrm{S}_{\mathrm{BDD}}, \mathrm{S}_{\mathrm{AIDM}}$.

By $K_{0}^{* L}$ - additional risk cancelation (Laplace) $\mathrm{S}_{\mathrm{WOA}}, \mathrm{S}_{\mathrm{CH}}$ are added to the recommendations, because these technologies are still at risk, even to only one.

(c) Инженерни науки, год. LVI, 2019, № 411 (c) Engineering Sciences, LVI, 2019, No. 4 
TABLE 5. $\mathrm{R}_{\mathrm{L}}$ - Change in labor market

\begin{tabular}{|c|c|c|c|c|c|c|c|c|c|c|}
\hline & $K_{\delta}$ & $K_{\delta}^{L}$ & $K_{P}$ & $K_{C}$ & $K_{O}$ & $K_{P}^{L}$ & $K_{C}^{L}$ & $K_{O}^{L}$ & $K_{0}^{*}$ & $K_{0}^{* L}$ \\
\hline $\begin{array}{c}\mathrm{S}_{\mathrm{VNI}} \\
\mathrm{S}_{\mathrm{WI}} \\
\mathrm{S}_{\mathrm{IMT}}\end{array}$ & 0.00 & 0.25 & 0.25 & -0.25 & -0.50 & 0.68 & -0.18 & -0.62 & -2.50 & -4.08 \\
\hline $\mathrm{S}_{\mathrm{DP}}$ & 0.00 & 0.50 & 0.50 & -0.50 & -1.00 & 1.00 & 0.00 & -0.50 & -5.00 & -4.50 \\
\hline $\begin{array}{c}\mathrm{S}_{\mathrm{UC}} \\
\mathrm{S}_{\mathrm{SOP}} \\
\end{array}$ & 0.00 & 0.25 & 0.25 & -0.25 & -0.50 & 0.68 & -0.18 & -0.62 & -2.50 & -4.08 \\
\hline $\begin{array}{c}\mathrm{S}_{\mathrm{WOA}}, \\
\mathrm{S}_{\mathrm{CH}}\end{array}$ & 0.00 & 0.13 & 0.13 & -0.13 & -0.25 & 0.46 & -0.21 & -0.54 & -1.25 & -3.18 \\
\hline $\mathrm{S}_{\text {IOT }}$ & 1.00 & 0.25 & 1.75 & 0.25 & -0.50 & 0.68 & -0.18 & -0.62 & -6.50 & -4.08 \\
\hline $\mathrm{S}_{\mathrm{SC}}$ & 0.00 & 0.25 & 0.25 & -0.25 & -0.50 & 0.68 & -0.18 & -0.62 & -2.50 & -4.08 \\
\hline $\begin{array}{l}\mathrm{S}_{\mathrm{BDD}} \\
\mathrm{S}_{\mathrm{AIDM}} \\
\end{array}$ & 1.00 & 0.75 & 1.25 & 0.75 & 0.50 & 1.18 & 0.32 & -0.12 & -1.50 & -3.58 \\
\hline $\mathrm{S}_{\mathrm{DC}}$ & 1.00 & 0.50 & 1.50 & 0.50 & 0.00 & 1.00 & 0.00 & -0.50 & -4.00 & -4.50 \\
\hline $\mathrm{S}_{\text {AIWCJ }}$ & 1.00 & 0.25 & 1.75 & 0.25 & -0.50 & 0.68 & -0.18 & -0.62 & -6.50 & -4.08 \\
\hline$S_{R \& S}$ & 1.00 & 0.38 & 1.63 & 0.38 & -0.25 & 0.86 & -0.11 & -0.59 & -5.25 & -4.47 \\
\hline $\mathrm{S}_{\mathrm{SE}}$ & 1.00 & 0.63 & 1.38 & 0.63 & 0.25 & 1.11 & 0.14 & -0.34 & -2.75 & -4.22 \\
\hline $\mathrm{S}_{3 \mathrm{DP}}$ & 1.00 & 0.63 & 1.38 & 0.63 & 0.25 & 1.11 & 0.14 & -0.34 & -2.75 & -4.22 \\
\hline
\end{tabular}

TABLE $6 . \mathrm{R}_{\mathrm{P}}$ - Mental distraction

\begin{tabular}{|c|c|c|c|c|c|c|c|c|c|c|}
\hline & $K_{\delta}$ & $K_{\delta}^{L}$ & $K_{P}$ & $K_{C}$ & $K_{O}$ & $K_{P}^{L}$ & $K_{C}^{L}$ & $K_{O}^{L}$ & $K_{0}^{*}$ & $K_{0}^{* L}$ \\
\hline $\begin{array}{c}\mathrm{S}_{\mathrm{VNI}}, \\
\mathrm{S}_{\mathrm{WI}} \\
\mathrm{S}_{\mathrm{IMT}}\end{array}$ & $\mathbf{1 . 0 0}$ & 0.25 & $\mathbf{1 . 7 5}$ & $\mathbf{0 . 2 5}$ & -0.50 & 0.68 & -0.18 & -0.62 & -6.50 & -4.08 \\
\hline $\mathrm{S}_{\mathrm{DP}}$ & 0.00 & 0.50 & 0.50 & -0.50 & -1.00 & 1.00 & 0.00 & -0.50 & -5.00 & -4.50 \\
\hline $\begin{array}{c}\mathrm{S}_{\mathrm{UC}}, \\
\mathrm{S}_{\mathrm{SOP}}\end{array}$ & 0.00 & 0.25 & 0.25 & -0.25 & -0.50 & 0.68 & -0.18 & -0.62 & -2.50 & -4.08 \\
\hline $\begin{array}{c}\mathrm{S}_{\mathrm{WOA}}, \\
\mathrm{S}_{\mathrm{CH}}\end{array}$ & 0.00 & 0.13 & 0.13 & -0.13 & $-\mathbf{0 . 2 5}$ & 0.46 & -0.21 & -0.54 & $-\mathbf{1 . 2 5}$ & $-\mathbf{3 . 1 8}$ \\
\hline $\mathrm{S}_{\mathrm{IOT}}$ & 0.00 & 0.25 & 0.25 & -0.25 & -0.50 & 0.68 & -0.18 & -0.62 & -2.50 & -4.08 \\
\hline $\mathrm{S}_{\mathrm{SC}}$ & 0.00 & 0.25 & 0.25 & -0.25 & -0.50 & 0.68 & -0.18 & -0.62 & -2.50 & -4.08 \\
\hline $\begin{array}{c}\mathrm{S}_{\mathrm{BDD}}, \\
\mathrm{S}_{\mathrm{AIDM}}\end{array}$ & 0.00 & $\mathbf{0 . 7 5}$ & 0.75 & -0.75 & -1.50 & $\mathbf{1 . 1 8}$ & $\mathbf{0 . 3 2}$ & $-\mathbf{0 . 1 2}$ & -7.50 & -3.58 \\
\hline $\mathrm{S}_{\mathrm{DC}}$ & 0.00 & 0.50 & 0.50 & -0.50 & -1.00 & 1.00 & 0.00 & -0.50 & -5.00 & -4.50 \\
\hline $\mathrm{S}_{\mathrm{AIWCJ}}$ & 0.00 & 0.25 & 0.25 & -0.25 & -0.50 & 0.68 & -0.18 & -0.62 & -2.50 & -4.08 \\
\hline $\mathrm{S}_{\mathrm{R} \& \mathrm{~S}}$ & 0.00 & 0.38 & 0.38 & -0.38 & -0.75 & 0.86 & -0.11 & -0.59 & -3.75 & -4.47 \\
\hline $\mathrm{S}_{\mathrm{SE}}$ & 0.00 & 0.63 & 0.63 & -0.63 & -1.25 & 1.11 & 0.14 & -0.34 & -6.25 & -4.22 \\
\hline $\mathrm{S}_{3 \mathrm{DP}}$ & 0.00 & 0.63 & 0.63 & -0.63 & -1.25 & 1.11 & 0.14 & -0.34 & -6.25 & -4.22 \\
\hline
\end{tabular}

(c) Инженерни науки, год. LVI, 2019, № $4 \quad 12$ (c) Engineering Sciences, LVI, 2019, No. 4 


\section{6. $\mathbf{R}_{\mathbf{P}}-$ MENTAL DISTRACTION}

Category $\mathrm{R}_{\mathrm{P}}$ includes risks: Accidents, trauma from negative immersive experiences, increased addiction and escapism, increased distractions (i.e. attention deficit disorder), escapism and/or addiction.

The analysis of the results in Table 6 for the evaluation of $\mathrm{R}_{\mathrm{P}}$ shows that by $K_{\delta}$ again, a maximum score of 1 indicates the presence of this risk when using technology. Since DM is indifferent to the risk selected technologies are $\mathrm{S}_{\mathrm{VNI}}, \mathrm{S}_{\mathrm{WI}}, \mathrm{S}_{\mathrm{IMT}}$, which contain it:

- By $K_{P}$, the maximum score is 1.75 . The choice to avoid using technology is reduced to $\mathrm{S}_{\mathrm{VNI}}, \mathrm{S}_{\mathrm{WI}}, \mathrm{S}_{\mathrm{IMT}}$, which generally have two risks, one being $\mathrm{R}_{\mathrm{P}}$;

- By $K_{C}$ it is recommended to avoid $\mathrm{S}_{\mathrm{VNI}}, \mathrm{S}_{\mathrm{WI}}, \mathrm{S}_{\mathrm{IMT}}$;

- $K_{O}$ recommends avoiding the minimum exposed $\mathrm{S}_{\mathrm{WOA}}, \mathrm{S}_{\mathrm{CH}}$;

- There is a similar recommendation by $K_{0}^{* L}$;

- The analysis of the other experiments in Tables 7-11 is analogous.

\section{7. $\mathbf{R}_{\mathrm{M}}$ - MANIPULATION AND ECHO CAMERA}

Category $\mathrm{R}_{\mathrm{M}}$ includes risks: Potentially be triggered by disseminating inaccurate information (the need for reputation management), lack of transparency

TABLE 7. $\mathrm{R}_{\mathrm{M}}$ - Manipulation and echo camera

\begin{tabular}{|c|c|c|c|c|c|c|c|c|c|c|}
\hline & $K_{\delta}$ & $K_{\delta}^{L}$ & $K_{P}$ & $K_{C}$ & $K_{O}$ & $K_{P}^{L}$ & $K_{C}^{L}$ & $K_{O}^{L}$ & $K_{0}^{*}$ & $K_{0}^{* L}$ \\
\hline $\begin{array}{c}\mathrm{S}_{\mathrm{VNI}}, \\
\mathrm{S}_{\mathrm{WI}}, \\
\mathrm{S}_{I M T}\end{array}$ & 0.00 & 0.25 & 0.25 & -0.25 & -0.50 & 0.68 & -0.18 & -0.62 & -2.50 & -4.08 \\
\hline $\mathrm{S}_{\mathrm{DP}}$ & $\mathbf{1 . 0 0}$ & 0.50 & 1.50 & 0.50 & 0.00 & 1.00 & 0.00 & -0.50 & -4.00 & -4.50 \\
\hline $\begin{array}{c}\mathrm{S}_{\mathrm{UC}}, \\
\mathrm{S}_{S O P}\end{array}$ & $\mathbf{1 . 0 0}$ & 0.25 & $\mathbf{1 . 7 5}$ & 0.25 & -0.50 & 0.68 & -0.18 & -0.62 & -6.50 & -4.08 \\
\hline $\begin{array}{c}\mathrm{S}_{\mathrm{WOA}}, \\
\mathrm{S}_{\mathrm{CH}}\end{array}$ & 0.00 & 0.13 & 0.13 & -0.13 & -0.25 & 0.46 & -0.21 & -0.54 & $-\mathbf{1 . 2 5}$ & $-\mathbf{3 . 1 8}$ \\
\hline $\mathrm{S}_{\mathrm{IOT}}$ & $\mathbf{1 . 0 0}$ & 0.25 & $\mathbf{1 . 7 5}$ & 0.25 & -0.50 & 0.68 & -0.18 & -0.62 & -6.50 & -4.08 \\
\hline $\mathrm{S}_{\mathrm{SC}}$ & 0.00 & 0.25 & 0.25 & -0.25 & -0.50 & 0.68 & -0.18 & -0.62 & -2.50 & -4.08 \\
\hline $\begin{array}{c}\mathrm{S}_{\mathrm{BDD}}, \\
\mathrm{S}_{\mathrm{AIDM}}\end{array}$ & $\mathbf{1 . 0 0}$ & $\mathbf{0 . 7 5}$ & 1.25 & $\mathbf{0 . 7 5}$ & $\mathbf{0 . 5 0}$ & $\mathbf{1 . 1 8}$ & $\mathbf{0 . 3 2}$ & $-\mathbf{0 . 1 2}$ & -1.50 & -3.58 \\
\hline $\mathrm{S}_{\mathrm{DC}}$ & 0.00 & 0.50 & 0.50 & -0.50 & -1.00 & 1.00 & 0.00 & -0.50 & -5.00 & -4.50 \\
\hline $\mathrm{S}_{\mathrm{AIWCJ}}$ & 0.00 & 0.25 & 0.25 & -0.25 & -0.50 & 0.68 & -0.18 & -0.62 & -2.50 & -4.08 \\
\hline $\mathrm{S}_{\mathrm{R \& S}}$ & 0.00 & 0.38 & 0.38 & -0.38 & -0.75 & 0.86 & -0.11 & -0.59 & -3.75 & -4.47 \\
\hline $\mathrm{S}_{\mathrm{SE}}$ & $\mathbf{1 . 0 0}$ & 0.63 & 1.38 & 0.63 & 0.25 & 1.11 & 0.14 & -0.34 & -2.75 & -4.22 \\
\hline $\mathrm{S}_{3 \mathrm{DP}}$ & $\mathbf{1 . 0 0}$ & 0.63 & 1.38 & 0.63 & 0.25 & 1.11 & 0.14 & -0.34 & -2.75 & -4.22 \\
\hline
\end{tabular}

(c) Инженерни науки, год. LVI, 2019, № 4 
where individuals are not privy to information algorithms (for news/information), complexity and loss of control, trust (how to trust data?), "Falling foul of the algorithm", becoming incomprehensible, increased manipulation and echo chambers.

\section{8. $R_{F}-$ FRAGMENTATION}

Category $\mathrm{R}_{\mathrm{F}}$ includes risks: Political fragmentation, groupthink within interest groups and increased polarization, increased inequality, lobbying against automation (people not allowed to drive on freeways), legal structures for driving, battles over algorithms, walled gardens (i.e. limited environments, for authenticated users only) do not allow full access in some regions/countries.

TABLE $8 . \mathrm{R}_{\mathrm{F}}-$ Fragmentation

\begin{tabular}{|c|c|c|c|c|c|c|c|c|c|c|}
\hline & $K_{\delta}$ & $K_{\delta}^{L}$ & $K_{P}$ & $K_{C}$ & $K_{O}$ & $K_{P}^{L}$ & $K_{C}^{L}$ & $K_{O}^{L}$ & $K_{0}^{*}$ & $K_{0}^{* L}$ \\
\hline $\begin{array}{c}\mathrm{S}_{\mathrm{VNI}}, \\
\mathrm{S}_{\mathrm{WI}}, \\
\mathrm{S}_{\mathrm{IMT}}\end{array}$ & 0.00 & 0.25 & 0.25 & -0.25 & -0.50 & 0.68 & -0.18 & -0.62 & -2.50 & -4.08 \\
\hline $\mathrm{S}_{\mathrm{DP}}$ & $\mathbf{1 . 0 0}$ & 0.50 & 1.50 & 0.50 & 0.00 & 1.00 & 0.00 & -0.50 & -4.00 & -4.50 \\
\hline $\begin{array}{c}\mathrm{S}_{\mathrm{UC}}, \\
\mathrm{S}_{\mathrm{SOP}}\end{array}$ & $\mathbf{1 . 0 0}$ & 0.25 & $\mathbf{1 . 7 5}$ & 0.25 & -0.50 & 0.68 & -0.18 & -0.62 & -6.50 & -4.08 \\
\hline $\begin{array}{c}\mathrm{S}_{\mathrm{WOA}} \\
\mathrm{S}_{\mathrm{CH}}\end{array}$ & 0.00 & 0.13 & 0.13 & -0.13 & -0.25 & 0.46 & -0.21 & -0.54 & $-\mathbf{1 . 2 5}$ & $-\mathbf{3 . 1 8}$ \\
\hline $\mathrm{S}_{\mathrm{IOT}}$ & 0.00 & 0.25 & 0.25 & -0.25 & -0.50 & 0.68 & -0.18 & -0.62 & -2.50 & -4.08 \\
\hline $\mathrm{S}_{\mathrm{SC}}$ & 0.00 & 0.25 & 0.25 & -0.25 & -0.50 & 0.68 & -0.18 & -0.62 & -2.50 & -4.08 \\
\hline $\begin{array}{c}\mathrm{S}_{\mathrm{BDD}}, \\
\mathrm{S}_{\mathrm{AIDM}}\end{array}$ & $\mathbf{1 . 0 0}$ & $\mathbf{0 . 7 5}$ & 1.25 & $\mathbf{0 . 7 5}$ & $\mathbf{0 . 5 0}$ & $\mathbf{1 . 1 8}$ & $\mathbf{0 . 3 2}$ & $-\mathbf{0 . 1 2}$ & -1.50 & -3.58 \\
\hline $\mathrm{S}_{\mathrm{DC}}$ & $\mathbf{1 . 0 0}$ & 0.50 & 1.50 & 0.50 & 0.00 & 1.00 & 0.00 & -0.50 & -4.00 & -4.50 \\
\hline $\mathrm{S}_{\mathrm{AIWCJ}}$ & 0.00 & 0.25 & 0.25 & -0.25 & -0.50 & 0.68 & -0.18 & -0.62 & -2.50 & -4.08 \\
\hline $\mathrm{S}_{\mathrm{R} \& \mathrm{~S}}$ & 0.00 & 0.38 & 0.38 & -0.38 & -0.75 & 0.86 & -0.11 & -0.59 & -3.75 & -4.47 \\
\hline $\mathrm{S}_{\mathrm{SE}}$ & 0.00 & 0.63 & 0.63 & -0.63 & -1.25 & 1.11 & 0.14 & -0.34 & -6.25 & -4.22 \\
\hline $\mathrm{S}_{3 \mathrm{DP}}$ & 0.00 & 0.63 & 0.63 & -0.63 & -1.25 & 1.11 & 0.14 & -0.34 & -6.25 & -4.22 \\
\hline
\end{tabular}

\section{9. $\mathbf{R}_{\mathrm{A}}-\mathrm{RESPONSIBILITY} \mathrm{AND} \mathrm{ACCOUNTABILITY}$}

Category $\mathrm{R}_{\mathrm{A}}$ includes risks: Formulas for specifying accountability (who owns the algorithm?), liability and accountability, governance, accountability (who is responsible, fiduciary rights), change to legal, financial disclosure, risk, decreased ability to measure this potentially grey economy.

(c) Инженерни науки, год. LVI, 2019, № 414 (c) Engineering Sciences, LVI, 2019, No. 4 
TABLE $9 . \mathrm{R}_{\mathrm{A}}$ - Responsibility and accountability

\begin{tabular}{|c|c|c|c|c|c|c|c|c|c|c|}
\hline & $K_{\delta}$ & $K_{\delta}^{L}$ & $K_{P}$ & $K_{C}$ & $K_{O}$ & $K_{P}^{L}$ & $K_{C}^{L}$ & $K_{O}^{L}$ & $K_{0}^{*}$ & $K_{0}^{* L}$ \\
\hline $\begin{array}{c}\mathrm{S}_{\mathrm{VNI}}, \\
\mathrm{S}_{\mathrm{WI}}, \\
\mathrm{S}_{\mathrm{IMT}}\end{array}$ & 0.00 & 0.25 & 0.25 & -0.25 & -0.50 & 0.68 & -0.18 & -0.62 & -2.50 & -4.08 \\
\hline $\mathrm{S}_{\mathrm{DP}}$ & 0.00 & 0.50 & 0.50 & -0.50 & -1.00 & 1.00 & 0.00 & -0.50 & -5.00 & -4.50 \\
\hline $\begin{array}{c}\mathrm{S}_{\mathrm{UC}}, \\
\mathrm{S}_{\mathrm{SOP}}\end{array}$ & 0.00 & 0.25 & 0.25 & -0.25 & -0.50 & 0.68 & -0.18 & -0.62 & -2.50 & -4.08 \\
\hline $\begin{array}{c}\mathrm{S}_{\mathrm{WOA}}, \\
\mathrm{S}_{\mathrm{CH}}\end{array}$ & 0.00 & 0.13 & 0.13 & -0.13 & -0.25 & 0.46 & -0.21 & -0.54 & $-\mathbf{1 . 2 5}$ & $-\mathbf{3 . 1 8}$ \\
\hline $\mathrm{S}_{\mathrm{IOT}}$ & 0.00 & 0.25 & 0.25 & -0.25 & -0.50 & 0.68 & -0.18 & -0.62 & -2.50 & -4.08 \\
\hline $\mathrm{S}_{\mathrm{SC}}$ & 0.00 & 0.25 & 0.25 & -0.25 & -0.50 & 0.68 & -0.18 & -0.62 & -2.50 & -4.08 \\
\hline $\begin{array}{c}\mathrm{S}_{\mathrm{BDD}}, \\
\mathrm{S}_{\mathrm{AIDM}}\end{array}$ & $\mathbf{1 . 0 0}$ & $\mathbf{0 . 7 5}$ & 1.25 & $\mathbf{0 . 7 5}$ & $\mathbf{0 . 5 0}$ & $\mathbf{1 . 1 8}$ & $\mathbf{0 . 3 2}$ & $-\mathbf{0 . 1 2}$ & -1.50 & -3.58 \\
\hline $\mathrm{S}_{\mathrm{DC}}$ & 0.00 & 0.50 & 0.50 & -0.50 & -1.00 & 1.00 & 0.00 & -0.50 & -5.00 & -4.50 \\
\hline $\mathrm{S}_{\mathrm{AIWCJ}}$ & $\mathbf{1 . 0 0}$ & 0.25 & $\mathbf{1 . 7 5}$ & 0.25 & -0.50 & 0.68 & -0.18 & -0.62 & -6.50 & -4.08 \\
\hline $\mathrm{S}_{\mathrm{R} \& \mathrm{~S}}$ & $\mathbf{1 . 0 0}$ & 0.38 & 1.63 & 0.38 & -0.25 & 0.86 & -0.11 & -0.59 & -5.25 & -4.47 \\
\hline $\mathrm{S}_{\mathrm{SE}}$ & $\mathbf{1 . 0 0}$ & 0.63 & 1.38 & 0.63 & 0.25 & 1.11 & 0.14 & -0.34 & -2.75 & -4.22 \\
\hline $\mathrm{S}_{3 \mathrm{DP}}$ & 0.00 & 0.63 & 0.63 & -0.63 & -1.25 & 1.11 & 0.14 & -0.34 & -6.25 & -4.22 \\
\hline
\end{tabular}

\section{0. $R_{E}-$ ECOLOGY, ECOSYSTEMS AND ETHICS}

Category $\mathrm{R}_{\mathrm{E}}$ includes risks: Comprehensive impact and provoke risks associated with growth in waste for disposal, and further burden on the environment, impact on agriculture from printing food, perverted disincentives for health: If everything can be replaced, why live in a healthy way?, existential threat to humanity, gun control: opening opportunities for printing objects with high levels of abuse, brand and product quality, major disruption of production controls, consumer regulations, trade barriers, patents, taxes and other government restrictions and, the struggle to adapt, ethical debates stemming from the printing of body parts and bodies: Who will control the ability to produce them? Who will ensure the quality of the resulting organs? Uncontrolled or unregulated production of body parts, medical equipment or food, production of parts in the layer process that are anisotropic, i.e. their strength is not the same in all directions, which could limit the functionality of parts, risk of collapse (total black out) if the (energy) system fails.

(c) Инженерни науки, год. LVI, 2019, № $4 \quad 15$ (C) Engineering Sciences, LVI, 2019, No. 4 
TABLE 10. $\mathrm{R}_{\mathrm{E}}-$ Ecology, ecosystems and ethics

\begin{tabular}{|c|c|c|c|c|c|c|c|c|c|c|}
\hline & $K_{\delta}$ & $K_{\delta}^{L}$ & $K_{P}$ & $K_{C}$ & $K_{O}$ & $K_{P}^{L}$ & $K_{C}^{L}$ & $K_{O}^{L}$ & $K_{0}^{*}$ & $K_{0}^{* L}$ \\
\hline $\begin{array}{c}\mathrm{S}_{\mathrm{VNI}}, \\
\mathrm{S}_{\mathrm{WI}} \\
\mathrm{S}_{\mathrm{IMT}}\end{array}$ & 0.00 & 0.25 & 0.25 & -0.25 & -0.50 & 0.68 & -0.18 & -0.62 & -2.50 & -4.08 \\
\hline $\mathrm{S}_{\mathrm{DP}}$ & $\mathbf{1 . 0 0}$ & 0.50 & 1.50 & 0.50 & 0.00 & 1.00 & 0.00 & -0.50 & -4.00 & -4.50 \\
\hline $\begin{array}{c}\mathrm{S}_{\mathrm{UC}}, \\
\mathrm{S}_{\mathrm{SOP}}\end{array}$ & 0.00 & 0.25 & 0.25 & -0.25 & -0.50 & 0.68 & -0.18 & -0.62 & -2.50 & -4.08 \\
\hline $\begin{array}{c}\mathrm{S}_{\mathrm{WOA}}, \\
\mathrm{S}_{\mathrm{CH}}\end{array}$ & 0.00 & 0.13 & 0.13 & -0.13 & -0.25 & 0.46 & -0.21 & -0.54 & $-\mathbf{1 . 2 5}$ & $-\mathbf{3 . 1 8}$ \\
\hline $\mathrm{S}_{\mathrm{IOT}}$ & 0.00 & 0.25 & 0.25 & -0.25 & -0.50 & 0.68 & -0.18 & -0.62 & -2.50 & -4.08 \\
\hline $\mathrm{S}_{\mathrm{SC}}$ & $\mathbf{1 . 0 0}$ & 0.25 & $\mathbf{1 . 7 5}$ & 0.25 & -0.50 & 0.68 & -0.18 & -0.62 & -6.50 & -4.08 \\
\hline $\begin{array}{c}\mathrm{S}_{\mathrm{BDD}}, \\
\mathrm{S}_{\mathrm{AIDM}}\end{array}$ & $\mathbf{1 . 0 0}$ & $\mathbf{0 . 7 5}$ & 1.25 & $\mathbf{0 . 7 5}$ & $\mathbf{0 . 5 0}$ & $\mathbf{1 . 1 8}$ & $\mathbf{0 . 3 2}$ & $-\mathbf{0 . 1 2}$ & -1.50 & -3.58 \\
\hline $\mathrm{S}_{\mathrm{DC}}$ & 0.00 & 0.50 & 0.50 & -0.50 & -1.00 & 1.00 & 0.00 & -0.50 & -5.00 & -4.50 \\
\hline $\mathrm{S}_{\mathrm{AIWCJ}}$ & 0.00 & 0.25 & 0.25 & -0.25 & -0.50 & 0.68 & -0.18 & -0.62 & -2.50 & -4.08 \\
\hline $\mathrm{S}_{\mathrm{R} \& \mathrm{~S}}$ & 0.00 & 0.38 & 0.38 & -0.38 & -0.75 & 0.86 & -0.11 & -0.59 & -3.75 & -4.47 \\
\hline $\mathrm{S}_{\mathrm{SE}}$ & 0.00 & 0.63 & 0.63 & -0.63 & -1.25 & 1.11 & 0.14 & -0.34 & -6.25 & -4.22 \\
\hline $\mathrm{S}_{3 \mathrm{DP}}$ & $\mathbf{1 . 0 0}$ & 0.63 & 1.38 & 0.63 & 0.25 & 1.11 & 0.14 & -0.34 & -2.75 & -4.22 \\
\hline
\end{tabular}

\section{1. $R_{S}$ - CHANGE IN INCOME/COST STRUCTURE AND OWNERSHIP OF ASSETS}

Category $R_{\mathrm{S}}$ includes risks: Effects on the whole economic and social system and redistribution mechanisms associated with the risk of primacy of intellectual property as a source of value in productivity, less investment capital available in the system, decreased revenue from traffic infringements, upending of insurance and roadside assistance ("pay more to drive yourself"), less car ownership (see Table 11).

\section{OVERALL RISK ASSESSMENT}

The results given in Table 12 were obtained by using the weights of the risks. In this particular experiment, it should be considered that the raw data is only of the type there is a risk " 1 " and no risk " 0 ", which determines the relatively sustainable result:

Recommendation is to avoid $\mathrm{S}_{\mathrm{BDD}}, \mathrm{S}_{\mathrm{AIDM}}$ technologies, which are exposed to maximum number of risks used in this study and to avoid the relatively most risky $\mathrm{S}_{\mathrm{WOA}}, \mathrm{S}_{\mathrm{CH}}$ if desired for complete safety.

Table 13 shows a summary of maximum scores obtained in all nine experiments. According to the design of the experiments the results could be used

(c) Инженерни науки, год. LVI, 2019, № $4 \quad 16 \quad$ (c) Engineering Sciences, LVI, 2019, No. 4 
TABLE 11. $\mathrm{R}_{\mathrm{S}}$ - Change in income/cost structure and ownership of assets

\begin{tabular}{|c|c|c|c|c|c|c|c|c|c|c|}
\hline & $K_{\delta}$ & $K_{\delta}^{L}$ & $K_{P}$ & $K_{C}$ & $K_{O}$ & $K_{P}^{L}$ & $K_{C}^{L}$ & $K_{O}^{L}$ & $K_{0}^{*}$ & $K_{0}^{* L}$ \\
\hline $\begin{array}{c}\mathrm{S}_{\mathrm{VNI}}, \\
\mathrm{S}_{\mathrm{WI}} \\
\mathrm{S}_{\mathrm{IMT}}\end{array}$ & 0.00 & 0.25 & 0.25 & -0.25 & -0.50 & 0.68 & -0.18 & -0.62 & -2.50 & -4.08 \\
\hline $\mathrm{S}_{\mathrm{DP}}$ & 0.00 & 0.50 & 0.50 & -0.50 & -1.00 & 1.00 & 0.00 & -0.50 & -5.00 & -4.50 \\
\hline $\begin{array}{c}\mathrm{S}_{\mathrm{UC}}, \\
\mathrm{S}_{\mathrm{SOP}}\end{array}$ & 0.00 & 0.25 & 0.25 & -0.25 & -0.50 & 0.68 & -0.18 & -0.62 & -2.50 & -4.08 \\
\hline $\begin{array}{c}\mathrm{S}_{\mathrm{WOA}}, \\
\mathrm{S}_{\mathrm{CH}}\end{array}$ & 0.00 & 0.13 & 0.13 & -0.13 & -0.25 & 0.46 & -0.21 & -0.54 & $-\mathbf{1 . 2 5}$ & $-\mathbf{3 . 1 8}$ \\
\hline $\mathrm{S}_{\mathrm{IOT}}$ & 0.00 & 0.25 & 0.25 & -0.25 & -0.50 & 0.68 & -0.18 & -0.62 & -2.50 & -4.08 \\
\hline $\mathrm{S}_{\mathrm{SC}}$ & 0.00 & 0.25 & 0.25 & -0.25 & -0.50 & 0.68 & -0.18 & -0.62 & -2.50 & -4.08 \\
\hline $\begin{array}{c}\mathrm{S}_{\mathrm{BDD}}, \\
\mathrm{S}_{\mathrm{AIDM}}\end{array}$ & 0.00 & $\mathbf{0 . 7 5}$ & 0.75 & -0.75 & -1.50 & $\mathbf{1 . 1 8}$ & $\mathbf{0 . 3 2}$ & $-\mathbf{0 . 1 2}$ & -7.50 & -3.58 \\
\hline $\mathrm{S}_{\mathrm{DC}}$ & $\mathbf{1 . 0 0}$ & 0.50 & $\mathbf{1 . 5 0}$ & 0.50 & 0.00 & 1.00 & 0.00 & -0.50 & -4.00 & -4.50 \\
\hline $\mathrm{S}_{\mathrm{AIWCJ}}$ & 0.00 & 0.25 & 0.25 & -0.25 & -0.50 & 0.68 & -0.18 & -0.62 & -2.50 & -4.08 \\
\hline $\mathrm{S}_{\mathrm{R} \& \mathrm{~S}}$ & 0.00 & 0.38 & 0.38 & -0.38 & -0.75 & 0.86 & -0.11 & -0.59 & -3.75 & -4.47 \\
\hline $\mathrm{S}_{\mathrm{SE}}$ & $\mathbf{1 . 0 0}$ & 0.63 & 1.38 & $\mathbf{0 . 6 3}$ & $\mathbf{0 . 2 5}$ & 1.11 & 0.14 & -0.34 & -2.75 & -4.22 \\
\hline $\mathrm{S}_{3 \mathrm{DP}}$ & $\mathbf{1 . 0 0}$ & 0.63 & 1.38 & $\mathbf{0 . 6 3}$ & $\mathbf{0 . 2 5}$ & 1.11 & 0.14 & -0.34 & -2.75 & -4.22 \\
\hline
\end{tabular}

TABLE 12. Overall risk assessment

\begin{tabular}{|c|c|c|c|c|c|c|c|c|c|c|}
\hline & $K_{\delta}$ & $K_{\delta}^{L}$ & $K_{P}$ & $K_{C}$ & $K_{O}$ & $K_{P}^{L}$ & $K_{C}^{L}$ & $K_{O}^{L}$ & $K_{0}^{*}$ & $K_{0}^{* L}$ \\
\hline $\begin{array}{c}\mathrm{S}_{\mathrm{VNI}}, \\
\mathrm{S}_{\mathrm{WI}}, \\
\mathrm{S}_{\mathrm{IMT}}\end{array}$ & 0.23 & 0.25 & 0.65 & -0.19 & -0.61 & 0.68 & -0.18 & -0.62 & -3.98 & -4.08 \\
\hline $\mathrm{S}_{\mathrm{DP}}$ & 0.43 & 0.50 & 0.93 & -0.08 & -0.58 & 1.00 & 0.00 & -0.50 & -4.58 & -4.50 \\
\hline $\begin{array}{c}\mathrm{S}_{\mathrm{UC}}, \\
\mathrm{S}_{\mathrm{SOP}}\end{array}$ & 0.28 & 0.25 & 0.72 & -0.17 & -0.62 & 0.68 & -0.18 & -0.62 & -4.20 & -4.08 \\
\hline $\begin{array}{c}\mathrm{S}_{\mathrm{WOA}}, \\
\mathrm{S}_{\mathrm{CH}}\end{array}$ & 0.08 & 0.13 & 0.36 & -0.20 & -0.47 & 0.46 & -0.21 & -0.54 & $\mathbf{- 2 . 6 7}$ & $-\mathbf{3 . 1 8}$ \\
\hline $\mathrm{S}_{\mathrm{IOT}}$ & 0.25 & 0.25 & 0.68 & -0.18 & -0.62 & 0.68 & -0.18 & -0.62 & -4.08 & -4.08 \\
\hline $\mathrm{S}_{\mathrm{SC}}$ & 0.15 & 0.25 & 0.52 & -0.22 & -0.59 & 0.68 & -0.18 & -0.62 & -3.56 & -4.08 \\
\hline $\begin{array}{c}\mathrm{S}_{\mathrm{BDD}}, \\
\mathrm{S}_{\mathrm{AIDM}}\end{array}$ & $\mathbf{0 . 8 3}$ & $\mathbf{0 . 7 5}$ & $\mathbf{1 . 2 1}$ & $\mathbf{0 . 4 4}$ & $\mathbf{0 . 0 5}$ & $\mathbf{1 . 1 8}$ & $\mathbf{0 . 3 2}$ & $-\mathbf{0 . 1 2}$ & -3.05 & -3.58 \\
\hline $\mathrm{S}_{\mathrm{DC}}$ & 0.43 & 0.50 & 0.93 & -0.07 & -0.57 & 1.00 & 0.00 & -0.50 & -4.57 & -4.50 \\
\hline $\mathrm{S}_{\mathrm{AIWCJ}}$ & 0.40 & 0.25 & 0.91 & -0.11 & -0.62 & 0.68 & -0.18 & -0.62 & -4.72 & -4.08 \\
\hline $\mathrm{S}_{\mathrm{R} \& \mathrm{~S}}$ & 0.48 & 0.38 & 0.99 & -0.03 & -0.54 & 0.86 & -0.11 & -0.59 & -4.63 & -4.47 \\
\hline $\mathrm{S}_{\mathrm{SE}}$ & 0.61 & 0.63 & 1.09 & 0.12 & -0.37 & 1.11 & 0.14 & -0.34 & -4.29 & -4.22 \\
\hline $\mathrm{S}_{3 \mathrm{BP}}$ & 0.43 & 0.63 & 0.96 & -0.11 & -0.64 & 1.11 & 0.14 & -0.34 & -4.91 & -4.22 \\
\hline
\end{tabular}

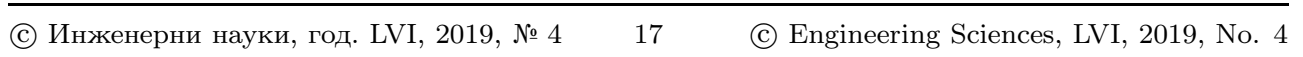


as help to identify riskiest technologies and give a recommendation for caution or rejection when the DM tightens the safety requirements.

TABLE 13

\begin{tabular}{|c|c|c|c|c|c|c|c|c|c|c|}
\hline Criteria & $K_{\delta}$ & $K_{P}$ & $K_{C}$ & $K_{O}$ & $K_{\delta}^{L}$ & $K_{P}^{L}$ & $K_{C}^{L}$ & $K_{O}^{L}$ & $K_{0}^{*}$ & $K_{0}^{* L}$ \\
\hline $\mathrm{R}_{\mathrm{D}}$ & 1.00 & 1.88 & 0.75 & 0.50 & 0.75 & 1.18 & 0.32 & -0.12 & -1.50 & -3.18 \\
\hline $\mathrm{R}_{\mathrm{L}}$ & 1.00 & 1.75 & 0.75 & 0.50 & 0.75 & 1.18 & 0.32 & -0.12 & -1.25 & -3.18 \\
\hline $\mathrm{R}_{\mathrm{P}}$ & 1.00 & 1.75 & 0.25 & -0.25 & 0.75 & 1.18 & 0.32 & -0.12 & -1.25 & -3.18 \\
\hline $\mathrm{R}_{\mathrm{M}}$ & 1.00 & 1.75 & 0.75 & 0.50 & 0.75 & 1.18 & 0.32 & -0.12 & -1.25 & -3.18 \\
\hline $\mathrm{R}_{\mathrm{F}}$ & 1.00 & 1.75 & 0.75 & 0.50 & 0.75 & 1.18 & 0.32 & -0.12 & -1.25 & -3.18 \\
\hline $\mathrm{R}_{\mathrm{A}}$ & 1.00 & 1.75 & 0.75 & 0.50 & 0.75 & 1.18 & 0.32 & -0.12 & -1.25 & -3.18 \\
\hline $\mathrm{R}_{\mathrm{E}}$ & 1.00 & 1.75 & 0.75 & 0.50 & 0.75 & 1.18 & 0.32 & -0.12 & -1.25 & -3.18 \\
\hline $\mathrm{R}_{\mathrm{S}}$ & 1.00 & 1.50 & 0.63 & 0.25 & 0.75 & 1.18 & 0.32 & -0.12 & -1.25 & -3.18 \\
\hline $\mathrm{R}_{\mathrm{D}} \mathrm{R}_{\mathrm{L}}$ & & & & & & & & & & \\
$\mathrm{R}_{\mathrm{M}} \mathrm{R}_{\mathrm{P}}$ & 0.83 & 1.21 & 0.44 & 0.05 & 0.75 & 1.18 & 0.32 & -0.12 & -2.67 & -3.18 \\
$\mathrm{R}_{\mathrm{F}} \mathrm{R}_{\mathrm{A}}$ & & & & & & & & & & \\
$\mathrm{R}_{\mathrm{E}} \mathrm{R}_{\mathrm{S}}$ & & & & & & & & & & \\
\hline
\end{tabular}

The results are an attempt to summarize the schematic picture that demonstrates the potential risks of using new IT. The presented interpretation can be used as a framework and starting point for further refined studies with a greater weight on the preferences of DM. Deeper analysis requires more additional and specific information not only about the quantitative dimensions of the risks, but also about the correlation and intersection of Its that inevitably exists. It is not only about new risks, but also at the level of technology use, because they are globally connected. The center, however, is in Big Data for Decisions $[3,5]$ and AI and Decision-Making [3, 4]. There are possible other risk combinations for the study, with an emphasis on factors such as psychological, political, socio-economic, demographic, technology-driven aspects.

\section{INSTEAD OF CONCLUSION}

The use of technologies is inevitable for at least two reasons: the process of increase in technology portfolios is very fast and the current economic model considers the information speed factor and the efficiency and profit factors as absolutely equivalents. "We are already devoting huge scientific and technical recourses to creating ever more capable AI systems, with very little thought devoted to what happens if we succeed. ... Another way to avoid the underlying issue is to assert that concerns about risks arise form ignorance" [9].

As J. Mantas states "We must exercise great care and responsibility as we develop AI, and ethical AI must become a global priority. When we do

(c) Инженерни науки, год. LVI, 2019, № 4 18 (c) Engineering Sciences, LVI, 2019, No. 4 
so, I am optimistic that we can steward it to enhance society and, in the process, help solve many of our most pressing problems. As we invest in AI, we must not forget to invest even more in "human intelligence" - in its most diverse and inclusive form". Future efforts will be directed towards exploring new technologies and risks, building practical risk analysis models, methods and algorithms, and seeking tools for design of conventional harmony in the realization of "artificial intelligence - natural intelligence" - the search for answer "The biggest step or the last step?" [10].

\section{REFERENCES}

[1] K. Schwab, The Fourth Industrial Revolution, Crown Publishing Group, New York (2017), ISBN: 978-5247-5886-8, eBook ISBN: 978-1-5247-5887-5

[2] A. Ross, The Industries of the Future, Simon \& Schuster, Reprint edition (February 7, 2017). ISBN-10: 1476753660, ISBN-13: 978-1476753669.

[3] G. ILIEva, Decision analysis for big data platform selection, Engineering Sciences (2019) LVI (2) 5-18, ISSN 1312-5702 (Print), ISSN 2603-3542 (Online).

[4] I. Radeva, Multicriteria Fuzzy Sets Application in Economic Clustering Problems, Cybernetics and Information Technologies (2017) 17 (3) 29-46, ISSN: 13119702 (Print), ISSN: 1314-4081 (Online).

[5] I. Popchev And D. Orozova, Towards Bid Data Analytics in the E-learning Space, Cybernetics and Information Technologies (2019) 19 (3) 16-24, ISSN: 1311-9702 (Print), ISSN: 1314-4081 (Online).

[6] "Deep Shift Technology Tipping Points and Societal Impact", Survey Report, World Economic Forum, September 2015, p. 44, http://www3.weforum.org/ docs/WEF_GAC15_Technological_Tipping_Points_report_2015.pdf.

[7] www.weforum.org, World Economic Forum, The Global Risks Report $201914^{\text {th }}$ Edition, p. 107.

[8] http://parallel.bas.bg/lcst/2016/01-2016-I_Popchev_1.pdf

[9] S. Russell, Many Experts Say We Shouldn't Worry About Super intelligent AI They're Wrong. - IEEE Spectrum, http://spectrum.ieee.org/computing/softwere/many-experts-say-weshouldntworry-about-superintelligent-ai-theyre-wrong

[10] J. Mantas, "The biggest step or the last step?" - Global Head of Strategy and Offerings, IBM Global Business Services, 02 Jul 2019.

(C) Инженерни науки, год. LVI, 2019, № 4 


\title{
АНАЛИЗ НА РИСКА - ИНСТРУМЕНТ ЗА ИЗБОР НА ТЕХНОЛОГИЯ
}

\author{
ИваН ПОПЧЕВ* И ИРИНА РАДЕВА \\ Институт по информационни и комуникационни технологии, \\ Бглгарска академия на науките, \\ ул. „Акад. Г. Бончев“, бл. 2, 1113 София, Бглгария, \\ e-mails: ipopchev@iit.bas.bg;iradeva@iit.bas.bg \\ Резюме. Статията е опит за обобщение на схематичната кар- \\ тина за анализ на отрицателните и неопределени въздействия \\ от внедряване на новите технологии, обединени във формула- \\ та Индустрия 4.0. Целта е идентифициране на рисковете от \\ използване на технологии, както и кои от тях са най-рискови. \\ Основните технологии са анализирани чрез техните неизвест- \\ ни и отрицателни въздействия. Рисковете са групирани в осем \\ обобщени категории, свързани с проблемите на използване на \\ лични и сигурност на данните, промяната на пазара на тру- \\ да, фрагментацията, отговорността и отчетността, екология- \\ та, екосистемите и етиката, промените в структурата на до- \\ ходите/разходите и собствеността върху активите. Технологи- \\ ите са анализирани и избрани с квази-многокритериален мо- \\ дел SIGMA. Получените резултати следва да се тълкуват като \\ рамка и отправна точка в по-задълбочено разбиране на при- \\ ложението и въздействието на технологиите. \\ Ключови думи: анализ на риска, квази многокритериален ана- \\ лиз, Индустрия 4.0 - технология, селекция.
}

Received October 29, 2019

(c) Инженерни науки, год. LVI, 2019, № 4 